\title{
Influence of Krypton Seeding on EU DEMO Operation with Lithium Divertor
}

\author{
M. Poradziński ${ }^{1}$ (1) - I. Ivanova-Stanik ${ }^{1}$ (1) - G. Pełka ${ }^{1} \cdot$ V. Pericoli Ridolfini ${ }^{1}$ (I) $\cdot$ R. Zagórski $^{2}$
}

Accepted: 9 January 2021 / Published online: 3 February 2021

(C) The Author(s) 2021

\begin{abstract}
DEMO reactor with liquid lithium divertor operation in presence of krypton seeding is analyzed. Integrated core-scrape-off layer-divertor code COREDIV is used. Sputtering model is revised and sputtering dependence on the divertor surface temperature fitted to the experimental data is added. It was found that $\mathrm{Kr}$ seeding is beneficial for DEMO operation. Plasma fuel dilution by lithium ions is reduced to the level of $1 \%$ for high level of $\mathrm{Kr}$ seeding and fusion power is comparable to the modelling results for argon or neon seeded DEMO with tungsten divertor. Simulations show that high confinement mode is possible in presence of $\mathrm{Kr}$ seeding (power across the separatrix stays above the L-H threshold).
\end{abstract}

Keywords DEMO $\cdot$ Liquid metal divertor $\cdot$ Impurity $\cdot$ Lithium $\cdot$ Numerical modelling

\section{Introduction}

Modelling of DEMO reactor with liquid lithium divertor indicates [1] that the goal of a divertor power load reduction may be achieved by the fuel ions dilution in the core and consequently the reduction of the fusion power. Effectively, DEMO operation with liquid lithium divertor is not excluded but results in lower fusion gain factor, $Q \lesssim 20$ compared to $Q \simeq 35$ in the case of tungsten divertor with additional neon or argon seeding [2]. In DEMO reactor with a tungsten divertor a significant part of the fusion power in the core is dissipated by means of radiation, what allows for reducing divertor heat load. The predicted fusion gain value in not seeded DEMO with tungsten divertor may be close to 40 . However, estimated value of the target power load in such a scenario is above the technological limit for a tungsten divertor. Seeding of medium $\mathrm{Z}$ impurities may improve power dissipation in the SOL but for the price of a lower fusion gain value. Contrary to tungsten divertor seeding of medium and high $\mathrm{Z}$

M. Poradziński

michal.poradzinski@ifpilm.pl

1 Institute of Plasma Physics and Laser Microfusion, Hery Str. 23, 01-497 Warsaw, Poland

2 National Centre For Nuclear Research (NCBJ), 05-400 Otwock, Poland impurities in DEMO with lithium divertor might be beneficial from the fusion gain point of view. They are able to dissipate power both in the core and in the SOL (see Fig. 1 for radiative capabilities of $\mathrm{Li}, \mathrm{Kr}$ and $\mathrm{W}$ ), reducing divertor power load and, in effect, decreasing lithium dilution. This feature however comes at a cost of higher risk of radiative collapse and loosing operation in a high confinement mode (H-mode).

The aim of this work is to analyze operation of a DEMO reactor with liquid lithium divertor in which cooling of the plasma is achieved by impurity seeding where $\mathrm{Kr}(\mathrm{Z}=36)$ is chosen as seeded gas. Sputtering model is revised and data verified thermal sputtering component is added in order to cover a range of divertor surface temperature from the melting point up to the level where evaporation is a main source of lithium. Conditions in the reactor core and scrape-off layer and their dependence on the $\mathrm{Kr}$ concentration are analyzed by means of the code COREDIV $[3,4]$. The paper is organized as follows, modelling approach is described in Sect. 2, sputtering and evaporation models are described in Sect. 3, results and discussion are presented in Sect. 4. 


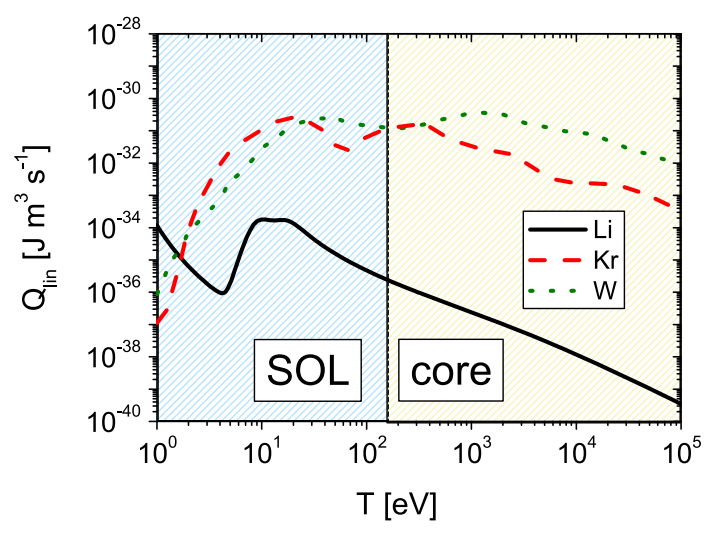

Fig. 1 Corona cooling rate

\section{Modelling Approach}

Modelling of fusion reactor plasma from the point of view of energy balance requires coupling between the core and the scrape-off layer (SOL) plasma. Intrinsic impurity sources like sputtering and evaporation depend on the particle and heat fluxes hitting the divertor plate. On the other hand presence of impurities in the core affects the fusion performance either by dilution (low $\mathrm{Z}$ impurities) or by additional radiation (medium and high $\mathrm{Z}$ impurities). The coupling between the core and the SOL requires the transport problem to be addressed in both regions simultaneously.

\section{COREDIV}

COREDIV is an integrated core-SOL-divertor code. The code self-consistently solves 1D radial transport equations of plasma and impurities in the core region and 2D multifluid transport based on Braginskii-like equations in the scrape-off layer region. Since this work is a continuation of work described in Ref. [1] we restrict here to listing the parameters used in the simulation and to the description of the sputtering model updated for the purpose of this study and refer the reader to Ref. [1] and references therein for more detailed description of the code. The value of radial anomalous transport in the SOL $D_{\text {rad }}^{S O L}=0.42 \mathrm{~m}^{2} / \mathrm{s}$. Thermal conductivities in the SOL are set to $\chi_{e, i}=0.18 \mathrm{~m}^{2} / \mathrm{s}$. Values for $D_{\text {rad }}^{S O L}$ and $\chi_{e, i}$ are chosen to be the same as in TECXY simulations for DEMO with liquid metal divertor [5]. Sputtering, prompt redeposition and evaporation of lithium is calculated self-consistently, depending on the plasma conditions in the divertor region. Since the evaporation model finds the liquid metal surface temperature it was straightforward to add to the physical sputtering module a component dependent on the target surface temperature.

\section{Lithium Source}

\section{Sputtering}

Physical sputtering model implemented in COREDIV is based on revised Bohdansky semi-empirical formula $[6,7]$ describing collisional sputtering on solid surfaces at normal incidence

$$
Y\left(E_{0}\right)=Q s_{n}^{K r C}(\varepsilon)\left(1-\left(\frac{E_{t h}}{E_{0}}\right)^{\frac{2}{3}}\right)\left(1-\frac{E_{t h}}{E_{0}}\right)^{2},
$$

where $s_{n}^{K r C}(\varepsilon)$ is the $\mathrm{Kr}-\mathrm{C}$ nuclear stopping cross section [8] and $\varepsilon$ is the reduced energy

$$
\varepsilon=E_{0} \frac{M_{2}}{M_{1}+M_{2}} \frac{a_{L}}{Z_{1} Z_{2} e^{2}} .
$$

$M_{1}\left(Z_{1}\right)$ and $M_{2}\left(Z_{2}\right)$ are atomic weights (atomic numbers) of the projectile and the target respectively and $a_{L}$ is the Lindhard screening length given by

$a_{L}=0.4685\left(Z_{1}^{2 / 3}+Z_{2}^{2 / 3}\right) \quad \AA$

and square of the electron charge $e^{2}=14.4 \mathrm{eV} \AA$. The values of the parameter $\mathrm{Q}$ and the thershold energy $E_{t h}$ for $\mathrm{D}+, \mathrm{He}+$, and $\mathrm{Li}+$ incident species are extracted from Ref. [9]. For other incident species like krypton the formula for the Q parameter and for the threshold energy is based on the formulas by Garcia-Rosales et al. [10].

A separate formula is used for the sputtering yield dependence on the angle of incidence $\alpha_{0}$ [11]

$$
\frac{Y\left(\alpha_{0}\right)}{Y(0)}=\frac{1}{\left(\cos \alpha_{0}\right)^{f}} \exp \left[\left(1-\frac{1}{\cos \alpha_{0}}\right) f \cos \alpha_{\text {opt }}\right] \text {, }
$$

where $f$ is a fitted parameter and $\alpha_{o p t}$ is the angle at which the sputtering yield reaches maximum. Values of parameters $f$ and $\alpha_{o p t}$ were calculted using formulas found in Ref. [11] with the exception of $\mathrm{D}+, \mathrm{He}+$ and $\mathrm{Li}+$ sputtering where the value of $f$ was extracted from Ref. [9]. Experiment by Allain at al. [9] in which the $\mathrm{D}+, \mathrm{He}+$ and $\mathrm{Li}+$ sputtering was measured were performed on D saturated $\mathrm{Li}$ target. In order to reproduce experimental results one has to include a model of preferential sputtering. Otherwise the sputtering yield calculated from formulas (1) and (4) provide overestimated sputtering yield values. Similarly as in Ref. [9] an expression derived by Andersen and Bay[12] was used

$$
Y_{c o l}=Y\left(E_{0}, \alpha_{0}\right) \frac{c_{L i}}{c_{D}}\left(\frac{M_{D}}{M_{L i}}\right)^{2 m}\left(\frac{U_{D}}{U_{L i}}\right)^{1-2 m},
$$

where $c_{D}$ and $c_{L i}$ are concentrations of $\mathrm{D}$ and $\mathrm{Li}$ atom on the lithium matrix and $U_{D}$ and $U_{L i}$ are the surface escape energy of each of the component. For simplicity it is 
assumed that $c_{L i}=c_{D}$ and $U_{D}=U_{L i}$ and only dependence on the masses is used. As for the parameter $m$ the value of $m=0.2$ is used. For He sputtering $M_{H e}$ is used instead of $M_{D}$. Although this might be an oversimplication of the expression (5) the results provide satisfying fit to the experimental data as can be seen in Fig. 2.

Sputtering on solid targets depends on the incident particle energy. However, experiments performed on liquid lithium targets showed strong sputtering dependence on the liquid metal surface temperature [13, 14]. In the considered DEMO divertor conditions the heat flux to the divertor target may heat the surface far above the melting point of the target but still below the point where evaporation is dominant. Therefore, it was necessary to include the thermal sputtering component along with the collisional one. In order to implement in COREDIV the sputtering dependence on the target surface temperature a function $Y_{t h}^{\text {fit }}(T)$ was added to the collisional sputtering yield

$Y_{\text {phys }}^{\text {exp } f i t}=Y_{\text {col }}\left(E_{0}\right) f\left(\alpha_{0}=45^{\circ}\right)+Y_{\text {th }}^{\text {fit }}$,

where $E_{0}=300 \mathrm{eV}, 500 \mathrm{eV}$ and $350 \mathrm{eV}$ for $\mathrm{D}+, \mathrm{He}+$ and $\mathrm{Li}+$ sputtering respectively. Formula from Eq. (4) is used for the angular dependence $f\left(\alpha_{0}\right)$. The thermal sputtering component dependence on the divertor surface temperature is based on the adatom model [15]

$Y_{t h}^{f i t}(T)=\frac{Y_{a d}}{1+A \exp \left(\frac{E_{e f f}}{k T}\right)}$

where the fitted parameters $Y_{a d}, A$ and $E_{\text {eff }}$ are listed in Table 1.

The fitted curves plotted against the experimental data are shown in Fig. 3. The solid black line corresponds to the self-sputtering and the solid red and blue lines correspond to helium and deuterium sputtering respectively.

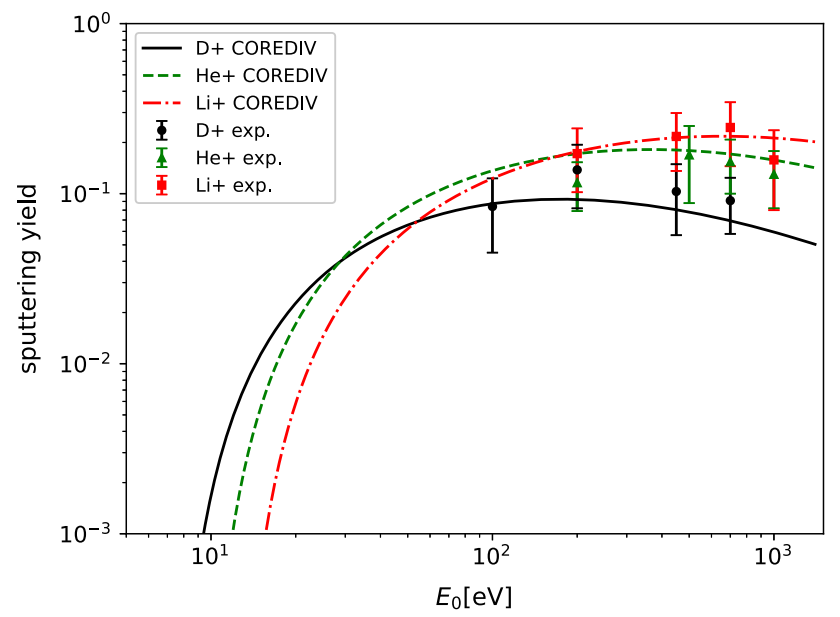

Fig. 2 Sputtering yield on solid lithium target at incident angle $\alpha_{0}=$ $45{ }^{\circ} \mathrm{C}$. Experimental data points by Allain et al. (Ref. [9])
Table 1 Fitted adatom model parameters for $\mathrm{D}+(300 \mathrm{eV}), \mathrm{He}+(500 \mathrm{eV})$ and $\mathrm{Li}+(350 \mathrm{eV})$ sputtering on $\mathrm{Li}$ target

\begin{tabular}{llll}
\hline & $\mathrm{D}+$ & $\mathrm{He}+$ & $\mathrm{Li}+$ \\
\hline$Y_{\text {ad }}$ & 0.646 & 1.06 & 2.7 \\
$\mathrm{~A}$ & $3.7 \times 10^{-4}$ & $4.27 \times 10^{-6}$ & $6.5 \times 10^{-6}$ \\
$E_{\text {eff }}[\mathrm{eV}]$ & 0.43 & 0.67 & 0.72 \\
\hline
\end{tabular}

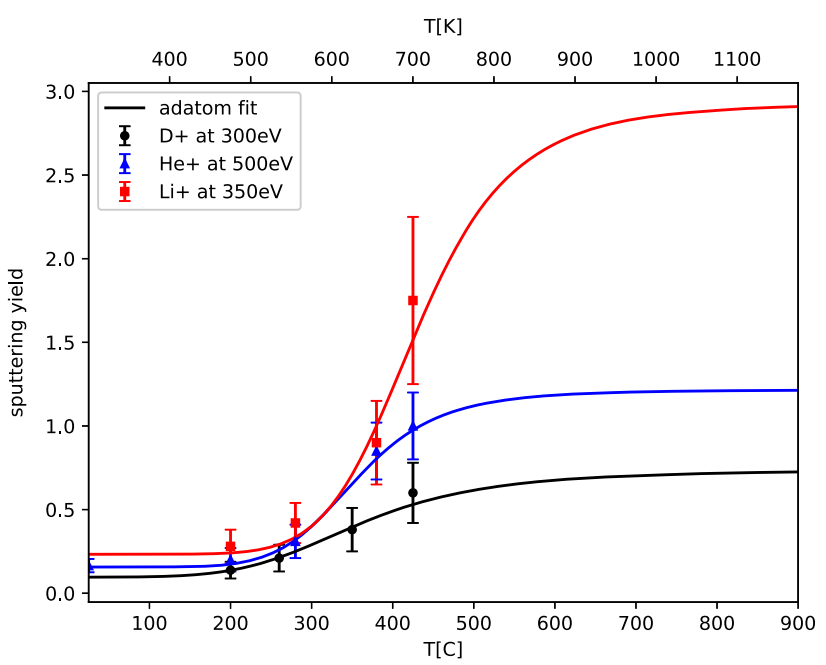

Fig. $3 \mathrm{D}+, \mathrm{He}+$ and self-sputtering dependence on temperature. Experimental data extracted from Refs. [13] and [14]

Since the measurement of thermal sputtering yield was done at the incindent angle $\alpha_{0}=45{ }^{\circ} \mathrm{C}$, throughout the simulation the same value of $\alpha_{0}$ was used. However, in order to facilitate the possiblity of angle dependent calculations in the future the available data was extrapolated to the values of the incident angle other than $45{ }^{\circ} \mathrm{C}$ using the following formula

$Y_{t h}(T)=0.33 \frac{\left.Y_{t h}^{f i t}(T)\right)}{f\left(45^{\circ}\right)}$

The dependence on the angle of incidence is factored out and the Li particle flux leaving the divertor plate can be written as

$$
\Gamma_{L i}=\Gamma_{\text {inc }} f\left(\alpha_{0}\right) Y_{\text {phys }}^{\text {normal }}+\Gamma_{\text {vap }},
$$

where $\Gamma_{i n c}$ is the flux of incident particles, $Y_{\text {phys }}^{\text {normal }}=$ $Y_{\text {col }}\left(E_{0}\right)+Y_{t h}(T)$ and $\Gamma_{\text {vap }}$ is the evaporation rate. Uisng this set of parameters the self-sputtering yield is less than unity with maximum value of $Y=0.97$ at $T=750 C{ }^{\circ} \mathrm{C}$ and $E_{0}=165 \mathrm{eV}$. 


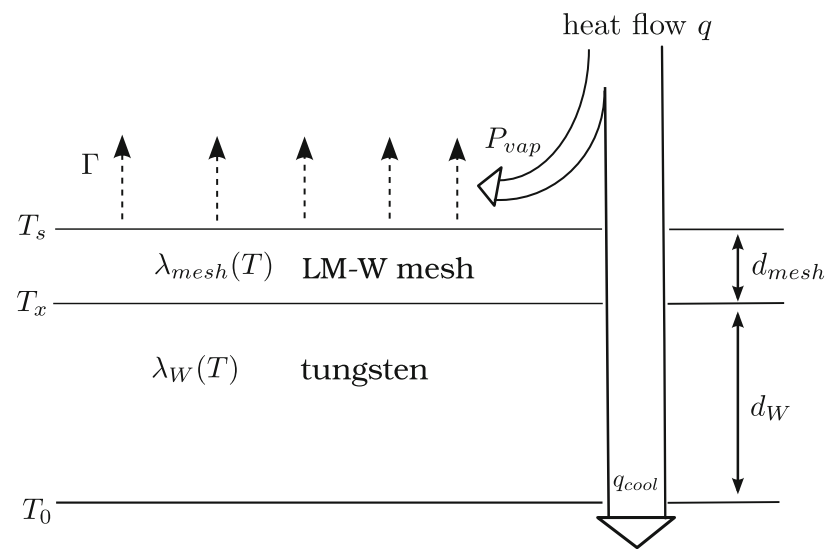

Fig. 4 Liquid metal divertor scheme

\section{Evaporation}

A one dimensional LM divertor scheme is presented in Fig. 4. The divertor consists of a metal mesh or 3-D printed structure wetted with liquid metal and a solid basis which is in contact with coolant. It is assumed that the heat flow across the divertor is one dimensional. For the purpose of this study, the metal mesh material and solid basis material is assumed to be tungsten. Temperatures $T_{x}$ and $T_{s}$ are the substrate-mesh contact surface temperature and the liquid metal surface temperature respectively. A liquid metal surface is assumed to be static. Heat flux $q$ is an input and evaporation rate $\Gamma$ is an output of the model. Parameters $d_{m e s h}$ and $d_{W}$ are a liquid metal mesh layer and a substrate layer thicknesses respectively. Lithium and tungsten thermal conductivities $\lambda_{i}(T), \mathrm{i}=\mathrm{Li}, \mathrm{W}$ for reference values at $800^{\circ} \mathrm{K}$ are given in Table 2 . Tungsten and lithium thermal conductivities dependence on temperature was extracted from Ref. [16]. Liquid metal and mesh thermal conductivity is an average of tungsten and lithium thermal conductivities. Coolant temperature $T_{0}$ is a parameter of the model and is kept constant during the simulations.

Heat flux $q$ is split into the heat flux $q_{\text {cool }}$ which is transferred without losses further to the bottom of the divertor plate and into latent heat of evaporation $P_{\text {vap }}$,

Table 2 Lithium and tungsten physical properties [16]

\begin{tabular}{lll}
\hline & $\mathrm{W}$ & $\mathrm{Li}$ \\
\hline Atomic number & 74 & 3 \\
Melting point, ${ }^{\circ} \mathrm{C}$ & 3422 & 180.5 \\
Boiling point, ${ }^{\circ} \mathrm{C}$ & 5930 & 1330 \\
Thermal conductivity at $800{ }^{\circ} \mathrm{K}, \mathrm{W} / \mathrm{m} / \mathrm{K}$ & 127.0 & 54.4 \\
Heat of evaporation, $\mathrm{kJ} / \mathrm{mol}$ & 806.7 & 136 \\
\hline
\end{tabular}

$q=q_{\text {cool }}+P_{\text {vap }}$.

Latent heat of evaporation is proportional to the evaporation rate $\Gamma, P_{\text {vap }}=h_{\text {vap }} \Gamma_{\text {vap }}$, where the coefficient $h_{\text {vap }}$ is a latent heat of evaporation per one particle. Temperatures $T_{x}$ and $T_{s}$ are the substrate-mesh contact surface temperature and the liquid metal surface temperature respectively. A flux of particles $\Gamma\left[\mathrm{m}^{-2} \mathrm{~s}^{-1}\right]$ leaving the divertor surface as a function of surface temperature is given by the HertzKnudsen equation.

$\Gamma_{v a p}=\frac{p_{s v}}{\sqrt{2 \pi k_{B} m_{L i} T_{s}}}$,

where saturated vapor pressure $p_{s v}=5.055-8023 / T_{s}$ [17]. The dependence of the evaporation rate on the liquid metal surface tempearature is close to the values presented in Refs. [18] and [19]. Surface temperature depends on the heat flux $q_{\text {cool }}$ reaching the divertor bottom via the Fourier equation $q_{\text {cool }}=-\lambda(T) \nabla T$. Eventually, the evaporation rate is calculated by solving Eq. (10), $\Gamma_{\text {vap }}=\left(q-q_{\text {cool }}\right) / h_{\text {vap }}$.

In Fig. 5 solid line corresponds to the solution of Eq. (10) and dashed line corresponds to the case when the second term $\left(P_{\text {vap }}\right)$ of the right hand side of Eq. (10) is neglected. Hence, the dashed line is the result of solving the Fourier equation with $q_{\text {cool }}$ equal to the heat flux q, which provides the surface temperature $T_{s}$ and applying directly the Hertz-Knudsen equation. The upper cut-off on the dashed line is set at the value of the heat flux where $T_{s}$ reaches the boiling point. At this point surface temperature reaches maximum and evaporation no longer increases. This is not the case when the latent heat of evaporation is included. Enthalpy of evaporation depends linearly on the evaporation rate, hence it becomes dominant at higher heat fluxes values and the evaporation rate exponential dependence on the heat flux gradually becomes linear with

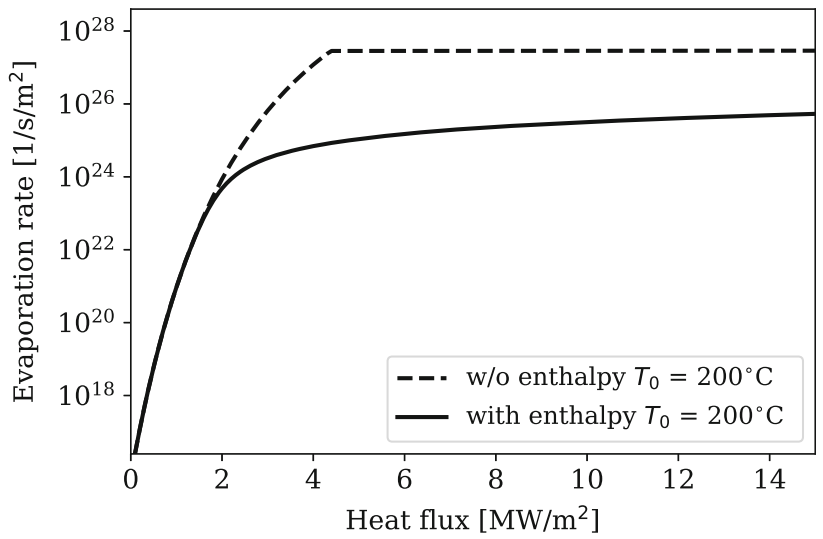

Fig. 5 Lithium evaporation rate dependence on the heat flux (solid) and the evaporation rate in the case when latent heat of evaporation is set to zero (dashed) 
asymptotic limit $\Gamma=q / h_{\text {vap }}$ and at the boiling point it becomes purely linear. This feature is responsible for saturation of the evaporation rate dependence on the heat flux allowing for higher fluxes and stabilizes the numerical behavior of the code.

\section{Modelling Results}

Simulations were performed according to the EU DEMO 2018 with the following main parameters: toroidal radius $R_{T}=9.002 \mathrm{~m}$, minor plasma radius $a=2.904 \mathrm{~m}$, plasma current $I_{p}=17.75 \mathrm{MA}$, toroidal magnetic field $B_{T}=5.855 \mathrm{~T}$, elongation $\varepsilon=1.65$, volume averaged electron density $\left\langle n_{e}\right\rangle_{\mathrm{VOL}}=7.261 \times 10^{19} \mathrm{~m}^{-3}$, L-H transition power threshold $P_{L H}=120.8 \mathrm{MW}, \quad \mathrm{H}$-factor (IPB98(y,2)) $H 98=1.1$ and auxiliary heating power $P_{\text {aux }}$ equal to $50 \mathrm{MW}$.

Throughout the simulatins the value of the density at the separatrix was set to $n_{\text {sep }}=3.27 \times 10^{19} \mathrm{~m}^{-3}$. The source of puffed impurity is located at 0.9 fraction of the distance from the stagnation point to divertor plate. It is a gaussian source with the half width of 0.05 (again as a fraction of the distance from the stagnation point to divertor plate). The $\mathrm{X}$-point is located at 0.83 of this distance, hence the puffing is applied already in the divertor region but closer to the Xpoint than to the divertor plate. Krypton as an inert gas is a highly recycling impurity and a position of source has minor influence on the SOL plasma. As for the evaporation model, the tungsten substrate depth was set to $d_{W}=10 \mathrm{~mm}$, liquid lithium mesh thickness $d_{L i}=1 \mathrm{~mm}$ and the temperature of the divertor bottom $T_{0}=200^{\circ} \mathrm{C}$.

\section{Kr Seeding}

$\mathrm{Kr}$ seeding was applied starting from the gas puff rate $\Gamma_{K r}^{p u f f}=10^{16} s^{-1}$ and it was increased up till the electron temperature at the plate stayed above $1 \mathrm{eV}$ or up till the code converged. Increasing krypton radiation in the core and in the SOL may eventually outbalance the heating energy which results in a radiational collapse in real conditions. In such a situation the corediv solver does not find the solution for the energy balance equation.

Dependence of impurities ( $\mathrm{Li}, \mathrm{He}$ and $\mathrm{Kr}$ ) concentrations in the core on the krypton gas puff rate is given in Fig. 6 (top). As expected Li concentration steadily decreases from $13.1 \%$ down to $1 \%$ of the total electron concentration. $\mathrm{Kr}$ concentration increases from practically zero to $0.134 \%$. Also $\mathrm{He}$ concentration increases as it's a product of increasing number of DT reactions. It is interesting to notice that the fuel dilution (Fig. 6, middle) in the center of the core defined as a ratio $\left(n_{e}^{0}-n_{i}^{0}\right) / n_{e}^{0}\left(n_{e, i}^{0}\right.$ being electron

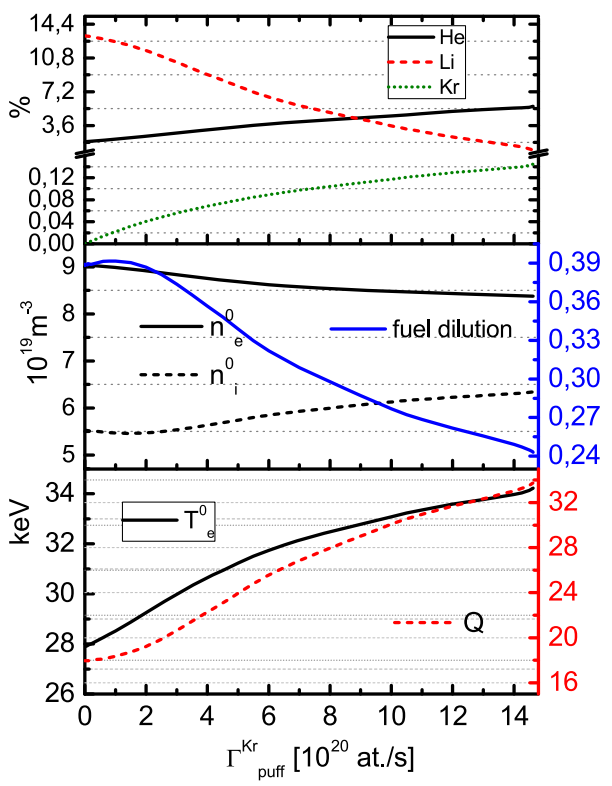

Fig. 6 Dependence on the $\mathrm{Kr}$ gas puff rate. Top: impurities concentration in the core. Bottom: electron temperature $T_{e}^{0}$ in the center of the core and fusion gain $\mathrm{Q}$. Middle: electron $\left(n_{e}^{0}\right)$ and main plasma ion $\left(n_{i}^{0}\right)$ density and fuel dilution in the center of the core

and ion concentration in the center of the core) increases first with the $\mathrm{Kr}$ gas puff rate reaching maximum at $\Gamma_{\text {puff }}^{K r} \simeq 10^{20} s^{-1}$. This is due to the fact that for small values of $\mathrm{Kr}$ gas puff ion density does not decrease as fast as electron density. The observed drop in electron density is mainly due to the fact that electron temperature in the center ( $T_{e}^{0}$, bottom of the Fig. 6) increases in result of the energy released in the increasing number of fusion reactions. Fusion gain is defined as $Q=5 P_{\alpha} / P_{a u x}$, where $P_{\alpha}$ is the energy carried by $\alpha$ particles and $P_{a u x}$ is the external heating power. Slight increase in fuel dilution for low values $\left(\Gamma_{\text {puff }}^{K r}<10^{20} s^{-1}\right)$ of the gas puff can explain why Q increases first slowly and from $\Gamma_{\text {puff }}^{K r} \simeq \times 10^{20} s^{-1}$ its slope becomes steeper and $\mathrm{Q}$ reaches 33.7 for the maximum gas puff level. The effect is more pronounce when $P_{\alpha}$ is plotted against the $\mathrm{Kr}$ concentration $\left(C_{K r}\right)$ in the core (see Fig. 7). Power balance in the core is shown in Fig. 7.Dashed green line $\left(P_{L H}\right)$ marks the minimum $P_{S O L}$ value necessary for the operation in the high confinement regime. At low level of $\mathrm{Kr}$ concentration radiation in the core increases faster than $\alpha$ power. This causes decrease of $P_{S O L}$ to the value of the L$\mathrm{H}$ transition. The value of $P_{S O L}$ reaches minimum of $114 \mathrm{MW}$ at $C_{K r}=0.055 \%$. At this point the slope of the heating power becomes steeper that the slope of core radiation curve and at higher level of $\mathrm{Kr}$ concentration $\left(C_{K r}>0.08 \%\right)$ power across the separatrix is higher than $\mathrm{L}-\mathrm{H}$ threshold.Changes in the power balance with krypton concentration are reflected in the increase of the radiation 


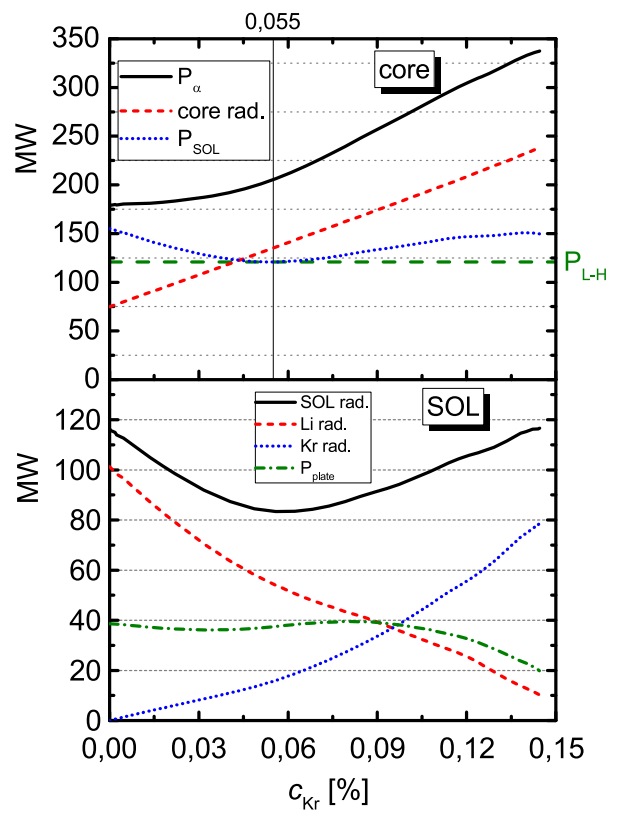

Fig. 7 Power balance dependence on the $\mathrm{Kr}$ concentration $\left(C_{K r}\right)$ in the core (top) and in the SOL (bottom)

fraction and the averaged effective charge $\left(Z_{\text {eff }}\right)$ (see Fig. 8.) Radiation fraction crosses the level of $90 \%$ and $Z_{\text {eff }}$ reaches 2.7 for the maximum value of $\mathrm{Kr}$ concentration. Changes in the power across the separatrix are balanced by radiation in the SOL keeping the total power flux to the divertor plates at the constant level of $\sim 30 \mathrm{MW}$ (see Fig. 7 bottom). Only for $C_{K r}>0.1 \%$ the total power to the plate starts to drop down below the level of 20MW for the maximum level of $\mathrm{Kr}$ concentration. Krypton radiation increases with the level of $\mathrm{Kr}$ concentration and partially replaces $\mathrm{Li}$ as a main radiator in the $\mathrm{SOL}$.

In the case of no $\mathrm{Kr}$ seeding lithium radiates ( see Fig. 9, dashed) predominantly directly in front of the divertor plate. When $\mathrm{Kr}$ seeding is applied the spike is replaced by a broader and smaller profile of $\mathrm{Kr}$ radiation (red) located

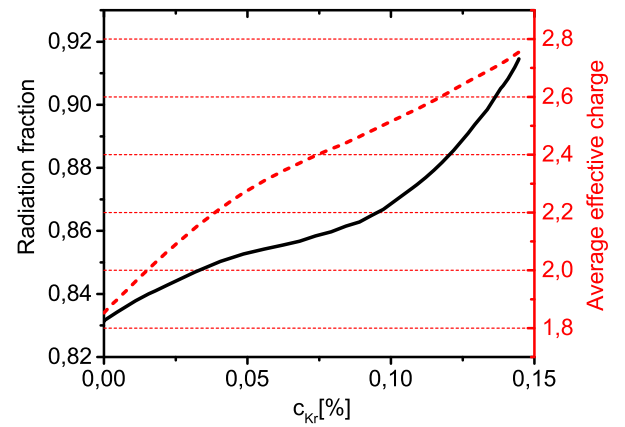

Fig. 8 Radiation fraction (solid line) and average effective charge (dashed) dependence on the $\mathrm{Kr}$ concetration in the core

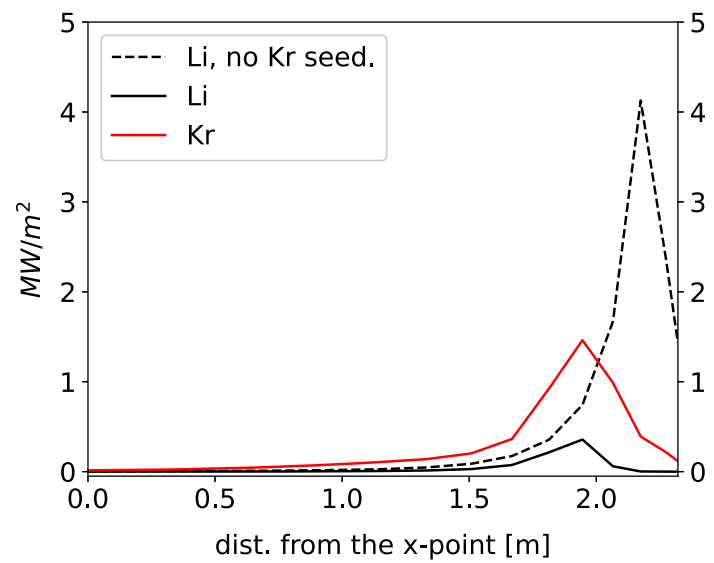

Fig. 9 Radiation profiles integrated along the radial direction of $\mathrm{Li}$ (black) and $\mathrm{Kr}$ (red) at $c_{K r}=0.14 \%$ and $\mathrm{Li}$ (dashed,no $\mathrm{Kr}$ seeding) as a function of distance from the $\mathrm{x}$-point. Divertor is located at the right border of the figure at the distance of $2.32 \mathrm{~m}$ from the x-point

further from the divertor plate. Li radiation (black, solid) is becomes much weaker and its maximum is aligned with the $\mathrm{Kr}$ radiation one.

As was observed in [1] evaporation was the dominant source of lithium in the plasma. However, when thermal sputtering is included, self-sputtering and evaporation contribute equally at low level of $\mathrm{Kr}$ concentration (see Fig. 10). Deuterium sputtering is order of magnitude lower than self-sputtering. Level of the $\mathrm{Li}$ influx rate $\left(\Gamma_{L i} \simeq 2 \times 10^{24} s^{-1}\right)$ and the maximum surface temperature $\left(T_{\text {surf }}^{\max }>750^{\circ} \mathrm{C}\right.$ ) found in the simulations (at low $\mathrm{Kr}$ seeding values) are comparable to those where thermal

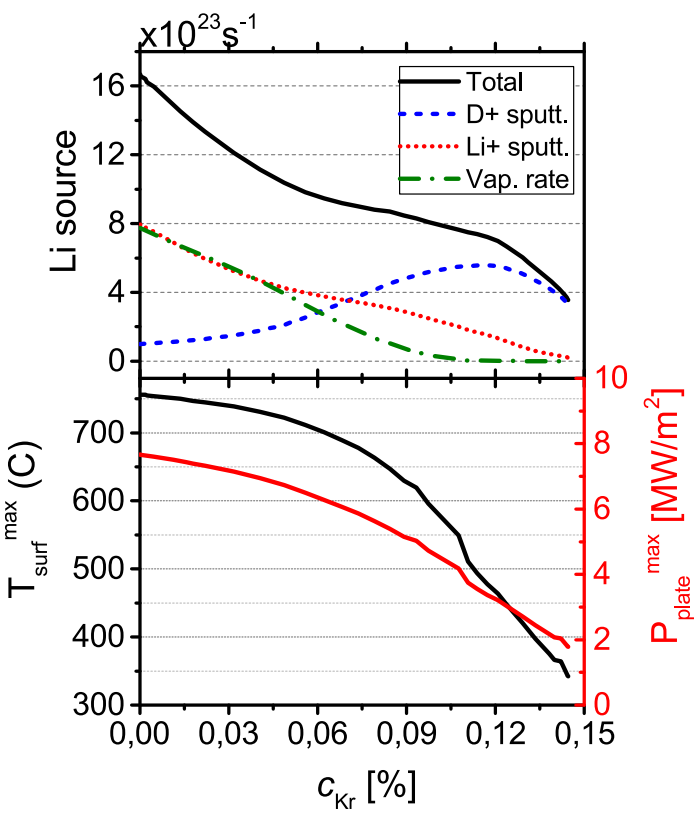

Fig. 10 Power balance dependence on the $\mathrm{Kr}$ concentration $\left(C_{K r}\right)$ in the core (top) and in the SOL (bottom) 
sputtering was neglected (see Ref. [1]). The noticable difference is that the plasma conditions in the divertor region at low $\mathrm{Kr}$ seeding found in the simulations when thermal sputtering is included correspond to the conditions found in Ref. [1] for higher values of tungsten substrate depth $\left(d_{W} \simeq 15-20 \mathrm{~mm}\right)$ rather than $d_{W}=10 \mathrm{~mm}$ used in this paper. One may conclude that including thermal sputtering results in high evaporation regime taking place for lower values of the LMD tungsten substrate depth.

In the case of pure lithium DEMO reactor predicted value of the divertor surface tempearture (above $700^{\circ} \mathrm{C}$ ) is higher than the numbers reported for example by [18] and [19]. This can be mostly attributed to the size of the lithium source, which is of the order of $10^{24} s^{-1}$ and is possible due to the volume of DEMO reactor and due the level of fusion power which is not dissipated by means of other impurities (which is the subject of this paper). Note, that in presence of krypton seeding the total $\mathrm{Li}$ source is lower but not significantly and stays at the level of $5 \times 10^{23} \mathrm{~s}^{-1}$ despite the fact that the surface temperature drops down to values below $400{ }^{\circ} \mathrm{C}$. As it was already noted, in the case of high $\mathrm{Kr}$ seeding the dominant source of lithium is sputtering.

Although power to the plate drops only by $30 \%$ with krypton concentration, conditions in the divertor region changes significantly with $\mathrm{Kr}$ seeding. Maximum power flux to the plate $\left(P_{\text {plate }}^{\text {max }}\right)$ drops from above $7 \mathrm{MW} / \mathrm{m}^{2}$ down to the value of $2 \mathrm{MW} / \mathrm{m}^{2}$ ( see Fig. 10). Maximum divertor surface temperature $\left(T_{\text {surf }}^{\max }\right)$ also drops from $755^{\circ} \mathrm{C}$ down to $350^{\circ} \mathrm{C}$ which results in a decline of the evaporation rate by few orders of magnitude. The total lithium influx to the plasma decreases with $\mathrm{Kr}$ concentration by $80 \%$ down to values $\Gamma_{L i} \sim 3.6 \times 10^{23} s^{-1}$ for the maximum $C_{K r}$ value. The hierarchy of the lithium sources changes. Deuterium sputtering contribution increases and equals self-sputtering at $C_{K r}=0.075 \%$. Self-sputtering drops down to the level of $\sim 2 \times 10^{22} s^{-1}$ which is $6 \%$ fraction of $\mathrm{D}$ sputtering) Electron temperature at the plate $\left(T_{e}^{\text {plate }}\right)$ drops from $14 \mathrm{eV}$ down to the level of $2 \mathrm{eV}$ and ion concentration at the plate increases significantly marking reduction of the lithium source (see Fig. 11).Maximum $\mathrm{Kr}$ concentration achieved during the simulations was $C_{K r}=0.145 \%$ at $\Gamma_{K r}^{p u f f}=1.46 \times 10^{21} s^{-1}$. Any attempts to further increase the gas puffing ended up in a collapse of the run. This may indicate a partial thermal collapse of the edge plasma as observed in [19] and therefore set the upper limit on $\mathrm{Kr}$ impurity concentration. This is however a very rough estimate taking into account limitations of the model used in this work to model extreme conditions of the scrape-off layer plasma. Factors which may influence operation at temperatures close to divertor detachment such as shape of the divertor, pumping efficency and private region are not taken into account.

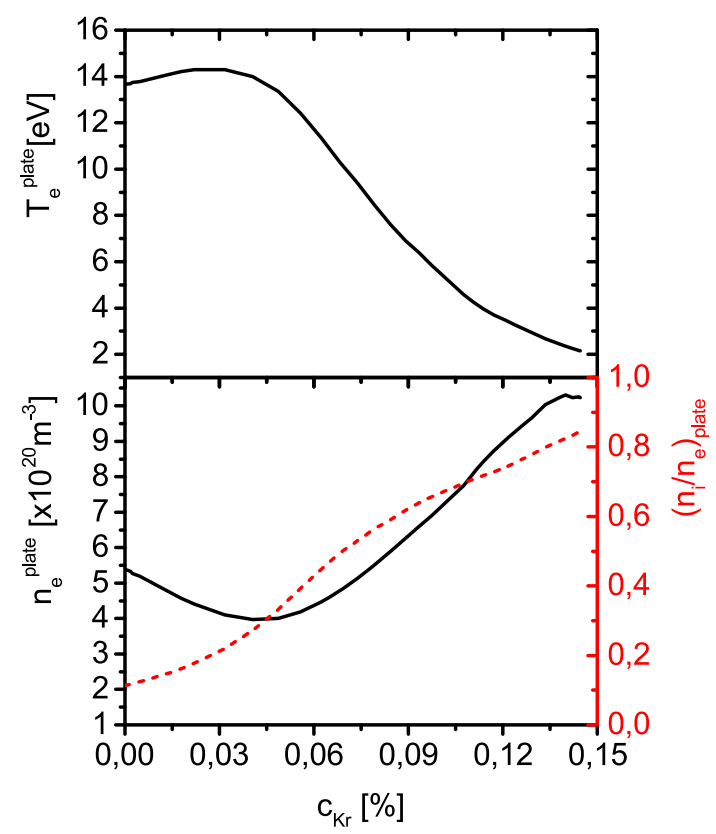

Fig. 11 Dependence on the $\mathrm{Kr}$ concentration. Top, electron temperature $\left(T_{e}^{\text {plate }}\right)$ at the strike point. Bottom, electron density $\left(n_{e}^{\text {plate }}\right)$ and ion density fraction $\left(\left(n_{i} / n_{e}\right)^{\text {plate }}\right)$ at the strike point

\section{Conclusions}

Lithium source model implemented in COREDIV was revised and thermal sputtering component ws added in order to stay in agreement with data available for sputtering on deuterium saturated lithium surface. Applying $\mathrm{Kr}$ seeding to a EU DEMO scenario with liquid lithium divertor helps in improving the power performance of the reactor. Fusion gain Q increases from values below 20 up the level above 30. Lithium dilution is significantly attenuated. Li concentration in the reactor core plasma drops from values above $13 \%$ down to around $1 \%$. Increasing fusion power balances krypton radiation in the core and power across the separatrix stays above the L-H threshold. However, in the mid values of $\mathrm{Kr}$ concentration $P_{S O L}$ stays only slightly above the L-H threshold. $\mathrm{Kr}$ seeding is also beneficial for the divertor power load. Maximum power load is kept at low level $\left(<8 \mathrm{MW} / \mathrm{m}^{2}\right)$ being reduced to $2 \mathrm{MW} / \mathrm{m}^{2}$ for the maximum krypton concentration level. Reduction in the divertor power load is accompanied by the decrease of the divertor surface temperature from the level of $70{ }^{\circ} \mathrm{C}$ to the level below $400^{\circ} \mathrm{C}$. Low temperature at the divertor plate and high level of SOL radiation for high values of $\mathrm{Kr}$ concentration may indicate that reactor operation at very high level of $\mathrm{Kr}$ seeding might be difficult to control.

Acknowledgements This work has been carried out within the framework of the EUROfusion Consortium and has received funding from the Euratom research and training programme 2014-2018 and 
2019-2020 under grant Agreement No 633053. The views and opinions expressed herein do not necessarily reflect those of the European Commission. This scientific work was partly supported by the Polish Ministry of Science and Higher Education within the framework of the scientific financial resources in the year 2020 allocated for the realization of the international co-financed Project No 5118/H2020/EURATOM/2020/2.

Open Access This article is licensed under a Creative Commons Attribution 4.0 International License, which permits use, sharing, adaptation, distribution and reproduction in any medium or format, as long as you give appropriate credit to the original author(s) and the source, provide a link to the Creative Commons licence, and indicate if changes were made. The images or other third party material in this article are included in the article's Creative Commons licence, unless indicated otherwise in a credit line to the material. If material is not included in the article's Creative Commons licence and your intended use is not permitted by statutory regulation or exceeds the permitted use, you will need to obtain permission directly from the copyright holder. To view a copy of this licence, visit http://creativecommons. org/licenses/by/4.0/.

\section{References}

1. M. Poradziński et al., Fusion Eng. Des. 146, 1500-1504 (2019)

2. I. Ivanova-Stanik et al., J. Nucl. Mater. 463, 596-600 (2014)

3. R. Zagórski et al., Contrib. Plasma Phys. 48(1-3), 179 (2008)

4. R. Zagórski et al., Nucl. Fusion 53, 073030 (2013)
5. V.P. Ridolfini et al., Phys. Plasmas 26, 012507 (2019). https://doi. org/10.1063/1.5055017

6. P. Sigmund, Phys. Rev.184, 383 (1969), errata: Phys. Rev.187, 768 (1969)

7. J. Bohdansky, H.L. Bay, J. Roth, J. Appl, Phys. 51, 2861 (1980). https://doi.org/10.1063/1.327954

8. W.D. Wilson, L.G. Haggmark, J.P. Biersack, Phys. Rev. B 15, 2458 (1977)

9. J.P. Allain, D.N. Ruzic, Nucl. Fusion 42, 202-210 (2002)

10. C. Garcia-Rosales et al., J. Nucl. Mater. 218, 8-17 (1994)

11. Y. Yamamura, Y. Itikawa, N. Itoh, Angular Dependence of Sputtering Yields of Monoatomic Solids Report IPPJ-AM-26 (Institute of Plasma Physics, Nagoya, 1983)

12. H.H. Andersen, H.L. Bay, Sputtering Yield by Particle Bombardment I, ed. by R. Behrisch, Springer, $1981 \mathrm{~S}$

13. J.P. Allain et al., Fusion Eng. Des. 72, 93-110 (2004)

14. J.P. Allain et al., Phys. Rev. B 76, 205434 (2007)

15. R.P. Doerner et al., J. Appl. Phys. 95, 4471 (2004). https://doi. org/10.1063/1.1687038

16. C.Y. Ho, R.W. Powell, P.E. Liley, J. Phys. Chem. Ref. Data 1, 279 (1972). https://doi.org/10.1063/1.3253100

17. C.B. Alcock, V.P. Itkin, M.K. Horrigan, Can. Metall. Q. 23(3), 309-313 (1984). https://doi.org/10.1179/cmq.1984.23.3.309

18. R.F. Mattas et al., Fusion Eng. Des. 49-50, 127-134 (2000)

19. T.D. Rognlien, M.E. Rensink, Phys. Plasmas 9, 2120 (2002)

Publisher's Note Springer Nature remains neutral with regard to jurisdictional claims in published maps and institutional affiliations. 